\title{
Magnetic Engine for the Single-Particle Landau Problem
}

\author{
Francisco J. Peña ${ }^{1, *}$, Alejandro González ${ }^{1}$, Alvaro S. Nunez ${ }^{2}$, Pedro A. Orellana ${ }^{1}$, \\ René G. Rojas ${ }^{3}$ and Patricio Vargas ${ }^{1,4}$ \\ 1 Departamento de Física, Universidad Técnica Federico Santa María, Valparaíso 2340000, Chile; \\ alephandros@gmail.com (A.G.); pedro.orellana.dinamarca@gmail.com (P.A.O.); \\ vargas.patricio@gmail.com (P.V.) \\ 2 Departamento de Física, Facultad de Ciencias Físicas y Matemáticas, Universidad de Chile, \\ Santiago 8320000, Chile; alvaro.sebastian.nunez@gmail.com \\ 3 Instituto de Física, Pontificia Universidad Católica de Valparaíso, Valparaíso 2340000, Chile; \\ rene.rojas.c@gmail.com \\ 4 Centro para el Desarrollo de la Nanociencia y la Nanotecnología, Santiago 8320000, Chile \\ * Correspondence: f.penarecabarren@gmail.com or francisco.penar@usm.cl
}

Received: 30 September 2017; Accepted: 22 November 2017; Published: 25 November 2017

\begin{abstract}
We study the effect of the degeneracy factor in the energy levels of the well-known Landau problem for a magnetic engine. The scheme of the cycle is composed of two adiabatic processes and two isomagnetic processes, driven by a quasi-static modulation of external magnetic field intensity. We derive the analytical expression of the relation between the magnetic field and temperature along the adiabatic process and, in particular, reproduce the expression for the efficiency as a function of the compression ratio.
\end{abstract}

Keywords: quantum thermodynamics; degeneracy effects; magnetic quantum engine

\section{Introduction}

Quantum thermodynamics is one of the most interesting topics in physics today. The possibility to create an alternative and efficient nanoscale device, like its macroscopic counterpart, introduces the concept of the quantum engine, which was proposed by Scovil and Schultz-Dubois in the 1950s [1]. The key point here is the quantum nature of the working substance and of course the quantum versions of the laws of thermodynamics [2-18]. The combination of these two simple facts leads to very interesting studies of well-known macroscopic engines of thermodynamics, such as Carnot, Stirling and Otto, among others [2-4].

The classical Otto engine consists of two isochoric processes and two adiabatic processes. If the working substance is a classical ideal gas, the first approximation for efficiency depends on the quotient of the temperatures in the first adiabatic compression [19,20]. This expression is reduced with the specific condition along the adiabatic trajectory for this kind of gas, given by $T V^{\gamma-1}=c n t$., where $\gamma=C_{P} / C_{V}$ obtaining the expression $\eta=1-\frac{1}{r^{\gamma-1}}$, where $r$ is defined as a "compression ratio" that is defined as $V_{1} / V_{2}$ (with $V_{1}>V_{2}$ ) [19]. On the other hand, the quantum Otto engine consists of two quantum adiabatic processes, which keeps invariant the probability occupation for the level of energy, and two quantum isochoric processes, in order to keep constant some parameters in the Hamiltonian. In this context, the quantum harmonic Otto cycle is a hot research topic [21-24], fully addressed by Kosloff and Rezek [25]. In addition, a recent experimental result has been achieved, employing a single ion confined in a linear Paul trap [26]. This research shed lights for a possible realization of a quantum Otto cycle. In the magnetic scenario, it is useful to think that the "isochoric processes" are replaced by "isomagnetic" ones where the constant value of the field in these 
strokes imply keeping constant value of the cyclotron frequency (or effective frequency depending on the case) due to the proportional relation between both quantities. This kind of approach is developed in the reference [13] for the case of a graphene under strain in the presence of an external magnetic field, exhibiting that the Carnot efficiency is achieved more quickly with the combination of these two effects as opposed to only applying strain to the sample.

The Landau levels of energy in condensed matter physics constitute a very well-known case and a typical academic problem. The thermodynamics is fully addressed in the works of Kumar et al. [27] where one important point is the degeneracy factor present in the partition function, and consequently also in the entropy.

In this way, a thermodynamic cycle, where the magnetic field can be controlled along the adiabatic trajectories will lead to very interesting new results which can be contrasted with the harmonic case. On the other hand, the effects of the degeneracy of energy levels on the efficiency and power of an engine quantum machine have been reported in many works in the past $[21,22,28-30]$. In this same framework, we highlight the work of Mehta and Ramandeep [31], who worked on a quantum Otto engine in the presence of level degeneracy, finding an enhancement of work and efficiency for two-level particles with a degeneracy in the excited state. Also, Azimi et al. present the study of a quantum Otto engine operating with a working substance of a single phase multiferroic $\mathrm{LiCu}_{2} \mathrm{O}_{2}$ tunable by external electromagnetic fields [22] and is extended by Chotorlishvili et al. [21] under the implementation of shortcuts to adiabaticity, finding a reasonable out power for the proposal machine. Physically it is nowadays possible to confine electrons in 2D. For instance, quantum confinement can be achieved in semiconductor hererojunctions, such as GaAs and AlGaAs. At $T=300 \mathrm{~K}$, the band gap of GaAs is $1.43 \mathrm{eV}$ while it is $1.79 \mathrm{eV}$ for AlxGa1- $x$ As $(x=0.3)$. Thus, the electrons in GaAs are confined in a 1 -D potential well of length $\mathrm{L}$ in the $Z$-direction. Therefore, electrons are trapped in $2 \mathrm{D}$ space, where a magnetic field along $Z$-axis can be applied [32].

Consequently, the study of electrons under controllable external fields is a topic of growing interest today. This work proposes to study the novel-magnetic cycle presented in the work [13] for the Landau problem and to understand the role of the degeneracy factor along the cycle. In particular, we found an analytical dependence between the magnetic field and temperature along the adiabatic process, and we use these results to calculate the efficiency of this cycle. We compare this efficiency to the one corresponding to the harmonic trap with the same parametrization to study how strong is the influence of this factor on the results.

\section{Partition Function for the Single-Particle Landau Problem}

We consider the case for an electron with an effective mass $m^{*}$ and charge $e$ placed in a magnetic field, where the Hamiltonian of this problem working in the symmetric gauge leads to the known expression

$$
\hat{H}=\frac{1}{2 m^{*}}\left[\left(p_{x}-\frac{e B y}{2}\right)^{2}+\left(p_{y}+\frac{e x B}{2}\right)\right],
$$

and the corresponding Landau levels display the energy spectrum

$$
\mathcal{E}_{n}=\hbar \omega_{B}\left(n+\frac{1}{2}\right)
$$

Here, $n=0,1,2, \ldots$ is the quantum number, and

$$
\omega_{B}=\frac{e B}{m^{*}}
$$

is the standard definition for the cyclotron frequency $[12,13,27]$. With the definition of the parameter $\omega_{B}$, we can define the Landau radius that captures the effect of the intensity of the magnetic field, 
given by $l_{B}=\sqrt{\hbar /\left(m^{*} \omega_{B}\right)}$. The energy spectrum for each level is degenerate with a degeneracy $g(B)$ given by [27]

$$
g(B)=\frac{e B}{2 \pi \hbar} \mathcal{A},
$$

with $\mathcal{A}$ being the area of the box perpendicular to the magnetic field $B$. Therefore, with this approach it is straightforward to calculate the partition function to Landau problem, and it turns out to be

$$
Z=\frac{m^{*} \omega_{B} \mathcal{A}}{4 \pi \hbar} \operatorname{csch}\left(\frac{\beta \hbar \omega_{B}}{2}\right)
$$

which corresponds to standard partition function for a harmonic oscillator in the canonical ensemble, with a degeneracy for level equal to $g(B)$.

\section{Thermodynamics and Magnetic Engine}

\subsection{The First Law of Thermodynamics: A Microscopic Approach.}

The first law of quantum thermodynamics is fully addressed in many works [2-18] and gives us the possibility to explore different quantum cycles and compare them with the classical analogues. We will follow the treatment of $[29,33,34]$, where they conceive a sequence of quasi-static process that drives the subsystem along the sequence of equilibrium states. To derivate this law simply (in a microscopic approach), consider a system describe by a density operator $\hat{\rho}$ and a Hamiltonian with an explicit dependence of some parameter that we will call $\xi$ in a generic form [34]. So, you have a set of eigenvectors of $\hat{H}$ that satisfy the eigenvalue problem

$$
\hat{H}|n ; \xi\rangle=\mathcal{E}_{n}|n ; \xi\rangle
$$

where $n$ represents a set of indexes that label the spectrum of the Hamiltonian and $|n ; \xi\rangle$ constitutes the set of eigenvectors of $\hat{H}$. The variations of work and heat are in general defined as [29]

$$
\begin{aligned}
& \delta W=\operatorname{Tr}\{\hat{\rho} d \hat{H}\} \\
& \delta Q=\operatorname{Tr}\{\hat{H} d \hat{\rho}\}
\end{aligned}
$$

On the other hand, when the eigenstates of the system Hamiltonian are used as the basis, and the coupling between the system and the environment is weak, the system can be described by a canonical distribution determined by $\hat{H}$ and the density matrix is diagonal in that representation [29]. Therefore, the ensemble-average energy $E=\langle\hat{H}\rangle$ is reduced to

$$
E=\sum_{n} P_{n}(\xi) \mathcal{E}_{n}(\xi),
$$

for a given occupation distribution with probabilities $P_{n}(\xi)$ in the $n$th eigenstate. So, we can describe the system by the energy levels and states derived from the Hamiltonian [29]. The statistical ensemble just described can be submitted to an arbitrary quasi-static process, involving the modulation of the parameter $\xi$, and hence the ensemble average energy changes accordingly,

$$
\begin{gathered}
d E=\sum_{n}\left(\mathcal{E}_{n}(\xi) d P_{n}(\xi)+P_{n}(\xi) d \mathcal{E}_{n}(\xi)\right) \\
=\delta Q+\delta W .
\end{gathered}
$$

The last equation corresponds to the microscopic formulation of the first law of thermodynamics [2-18,25-27,31,34,35]. The first term in Equation (10) is associated with the energy exchange, while the second term represents the work done. That is, the work performed corresponds to the change in the eigenenergies $\mathcal{E}_{n}(\xi)$. This is in agreement with the fact that work can only be carried 
out through a change in generalized coordinates of the system, which in turn gives rise to a change in the eigenenergies $[9,10]$. A very important assumption for Equation (10) is very well described in [29], where it is mentioned that: "small changes in $\hat{H}$, which can be considered as a first-order perturbation, only shifts the energy levels of the systems and does not modify its eigenstates".

The usual expression for the entropy is given by the von Neumann form and in the eigenenergy basis can be rewritten as

$$
S(\xi)=-k_{B} \sum_{n} P_{n}(\xi) \ln \left[P_{n}(\xi)\right]
$$

where the coefficients $P_{n}(\xi)$ satisfy that $0 \leq P_{n}(\xi) \leq 1$ with the normalization condition

$$
\sum_{n} P_{n}(\xi)=1
$$

\subsection{Magnetic Engine}

As mentioned above, the result of the efficiency of the conventional Otto cycle can be written in the form that the results only depend on the quotient of the temperatures in the first adiabatic compression. By using the properties of the ideal gas, the efficiency can be rewritten as follows:

$$
\eta=1-\frac{1}{r^{\gamma-1}}
$$

where $\gamma=C_{P} / C_{V}$ is the quotient of the two specific heat (at constant pressure and at constant volume) and $r$ is known as "compression ratio" which is defined as $\frac{V_{1}}{V_{2}}$ (with $V_{1}>V_{2}$ ).

On the other hand, the quantum "conventional" Otto engine is composed of two quantum isochoric processes and two quantum adiabatic processes. For the first mentioned process, the occupation probabilities $P_{n}(\xi)$ change and thus the entropy $S$ changes, until the working substance finally reaches thermal equilibrium with the heat bath. For the case of the quantum adiabatic process, the population distributions remain unchanged, that is $d P_{n}(\xi)=0$. Thus, no transition occurs between levels, and no heat is exchanged during this process. It is important to recall that in a classical adiabatic process the occupation of each level is never invariant (unless the classical thermalization condition is relaxed) [35]. This is a crucial point in the discussion for our magnetic engine, based on the work presented [13]. In general, a quantum adiabatic stroke does not maintain the system in a thermal state [28-30]. A power law potential can guarantee the thermal state condition when the degeneracy does not change, as in the treatment of [36], but not in our case under the study, because the number of states involved in the process should not increase, even if the degeneracy increases. So, if the cycle is strictly quantum, the stage after the quantum adiabatic stroke necessarily is a non-equilibrium state (only in the degenerate case), and cannot be represented for the partition function of Equation (5). Therefore, our approach uses the condition of a classical thermodynamic adiabaticity, where the process is identified in terms of the conservation of the entropy and the isolation of the system from heat exchange with the thermal bath. We recall that we work in a semi-classical scenario where the quantum part is related to the nature of the working substance and the classical part is due to the condition imposed over the adiabatic strokes along the cycle that we propose.

The case of conventional quantum Otto engine has been considered in several works for differents quantum systems $[3,9,13,31,36]$ where the key findings are an expression for the efficiency present in Equation (13) and establishing the value of $\gamma$ for that case. For the magnetic case, the two isochoric trajectories are replaced by two "isomagnetic" ones, in which the magnetic field intensity along the process remains constant while heat is exchanged between the system and the reservoirs [13].

Let us consider a cycle by devising a sequence of quasi-static trajectories, as depicted in Figure 1. First, the system, while submitted to an external magnetic field $B_{1}$, is brought into thermal equilibrium with macroscopic thermostats at temperature $T_{1}$. In equilibrium, the probabilities $P_{n}(\xi)$ take the Boltzmann form and can work with a partition function in the canonical ensemble, $Z(\xi, T)$. So, the Helmholtz free energy can be defined by $F(\xi, T)=-k_{B} T \ln Z(\xi, B)$ and the entropy given by 
Equation (11) can be written as $S(\xi, T)=\frac{E(\xi, T)}{T}+k_{B} \ln Z(\xi, T)$, where the ensemble average energy is given by

$$
E(\xi, T)=k_{B} T^{2} \frac{\partial}{\partial T} \ln Z(\xi, T) .
$$

Here, the $\xi$ parameter is related to the intensity of the magnetic field, so $\xi \rightarrow B$ for the case under the study. Then, the system is submitted to a quantum isoentropic process from $1 \rightarrow 2$, increasing the magnitude of the magnetic field from $B_{1}$ to $B_{2}$. The systems performs work along the isoentropic trajectory according to

$$
W_{1 \rightarrow 2}=\int_{B_{1}}^{B_{2}} d B\left(\frac{\partial E}{\partial B}\right)_{S}=E\left(T_{2}, B_{2}\right)-E\left(T_{1}, B_{1}\right) .
$$

For the case of the "isomagnetic" heating process with the intensity of magnetic field equal to $B_{2}$ from $2 \rightarrow 3$, no work is done, but heat is absorbed. The heat absorbed $\left(Q_{2 \rightarrow 3}\right)$ is given by the expression

$$
Q_{2 \rightarrow 3}=\int_{T_{2}}^{T_{3}} d T\left(\frac{\partial E}{\partial T}\right)_{B_{2}}=E\left(T_{3}, B_{2}\right)-E\left(T_{2}, B_{2}\right) .
$$

In the same way discussed before, the isoentropic trajectory from $3 \rightarrow 4$, the system perform work in the form

$$
W_{3 \rightarrow 4}=E\left(T_{4}, B_{1}\right)-E\left(T_{3}, B_{2}\right) .
$$

A physical interpretation of the work performed by the engine is obtained by considering the statistical mechanical definition of the ensemble-average magnetization, $M=-\left(\frac{\partial E}{\partial B}\right)_{S}$. Hence, the works defined in Equations (15) and (17) can also be interpreted as $W=-\int M d B[12,13]$.

Similarly, we obtain the heat released to the low temperature sink in the quantum "isomagnetic" cooling process from $4 \rightarrow 1$

$$
Q_{4 \rightarrow 1}=\int_{T_{4}}^{T_{1}} d T\left(\frac{\partial E}{\partial T}\right)_{B_{1}}=E\left(T_{1}, B_{1}\right)-E\left(T_{4}, B_{1}\right) .
$$

The efficiency of the engine is then given by the expression

$$
\eta=\left|\frac{W_{1 \rightarrow 2}+W_{3 \rightarrow 4}}{Q_{H}}\right|=1-\left|\frac{E\left(T_{1}, B_{1}\right)-E\left(T_{4}, B_{1}\right)}{E\left(T_{3}, B_{2}\right)-E\left(T_{2}, B_{2}\right)}\right| .
$$

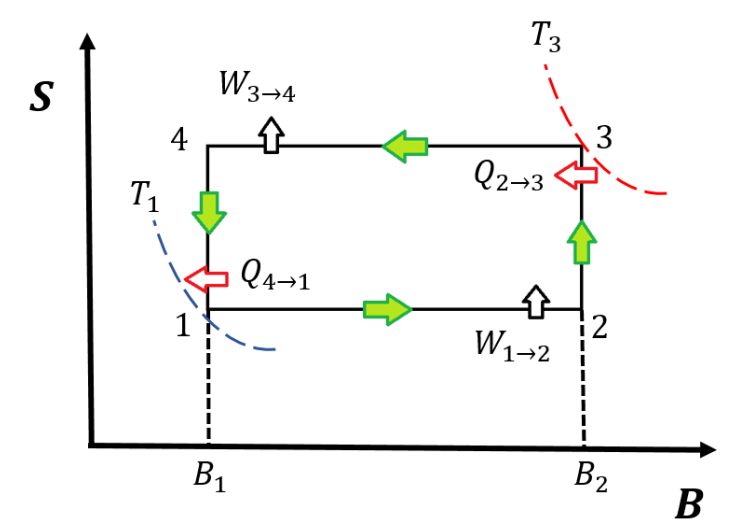

Figure 1. Pictorial description for the novel-magnetic engine represented as an entropy versus a magnetic field diagram. 
If we have the analytic function for the entropy, the intermediate temperatures $T_{2}$ and $T_{4}$ must be determined to reduce the expression for efficiency and can take on two different forms:

- Deducting the relation between the magnetic field and the temperature along an isoentropic trajectory solving the differential equation of first order given by

$$
d S(B, T)=\left(\frac{\partial S}{\partial B}\right)_{T} d B+\left(\frac{\partial S}{\partial T}\right)_{B} d T=0
$$

which can be written as

$$
\frac{d B}{d T}=-\frac{C_{B}}{T\left(\frac{\partial S}{\partial B}\right)_{T}},
$$

where $C_{B}$ is the specific heat at constant magnetic field.

- The other possibility is connecting the value for the entropy in two isoentropic trajectories in the form

$$
\begin{aligned}
& S\left(B_{1}, T_{1}\right)=S\left(B_{2}, T_{2}\right) \\
& S\left(B_{2}, T_{3}\right)=S\left(B_{1}, T_{4}\right),
\end{aligned}
$$

finding the function for the magnetic field in terms of the temperature through numerical calculation. Finally, we parametrize this dependency in the efficiency by defining the ratio

$$
r=\frac{l_{B_{1}}}{l_{B_{2}}}
$$

which represents the analogy of the compression ratio for the classical case. It is important to remember that the Landau radius is inversely proportional to the magnitude of the magnetic field. Therefore, for a major (minor) magnitude of the field, the Landau radius is smaller (bigger), and the $r$ parameter is well defined.

It is important to highlight the work of Zheng and Poletti [36], where they derived a general form for the efficiency of quantum Otto cycles with power law trapping potentials, corresponding to Equation (11), and showed that $\gamma$ must be equal to three. We remark that this result requires that two conditions are met. First, it is only valid for the non-degenerate cases, or more specifically, when the degeneracy is independent of the parameter that rules the cycle. The second condition is that the expansion process, when the system goes to $\omega^{\prime} \rightarrow \omega^{\prime \prime}$, must follow the following relation

$$
\kappa \equiv \frac{\mathcal{E}_{n}\left(\omega^{\prime}\right)}{\mathcal{E}_{n}\left(\omega^{\prime \prime}\right)}=\left(\frac{\omega^{\prime}}{\omega^{\prime \prime}}\right)^{\alpha}
$$

where $\alpha$ depends on the power of the potentials under study. For example, for a conventional harmonic trap, the spectrum of energy is always $\mathcal{E}_{n}=\hbar \omega\left(n+\frac{1}{2}\right)$ and we quickly obtain the result previously discussed. Moreover, this value of $\gamma$ is valid for a family of trapping potentials that fulfills the state equation $P V=2\langle E\rangle[36]$.

The case of the Landau problem is different. The energy spectrum of Equation (2) respects the condition of Equation (24) and has the structure of a harmonic trap; however, the degeneracy factor is a function of the magnetic field and the size of the system. Therefore, the results previously discussed do not hold, because in the first $(1 \rightarrow 2)$ and third $(3 \rightarrow 4)$ process, the change in the intensity of magnetic field leads to a change in the degeneracy factor, thus this problem must be analyzed carefully. 
Magnetic Engine for the Landau Problem

We show that the representation of the partition function for this case can be taken in the form of Equation (5). The thermodynamic quantities are present in the work of Kumar et al. [27], given by

$$
\begin{gathered}
\mathcal{F}=-\frac{1}{\beta} \ln \left[\frac{g(B)}{2} \operatorname{csch}\left(\frac{\beta \hbar \omega_{B}}{2}\right)\right], \\
E=\frac{\hbar \omega_{B}}{2} \operatorname{coth}\left(\frac{\beta \hbar \omega_{B}}{2}\right), \\
S_{L}=\frac{\hbar \omega_{B}}{2 T} \operatorname{coth}\left(\frac{\beta \hbar \omega_{B}}{2}\right)+k_{B} \ln \left[\frac{g(B)}{2} \operatorname{csch}\left(\frac{\beta \hbar \omega_{B}}{2}\right)\right],
\end{gathered}
$$

and the specific heat

$$
C_{B}=k_{B} \beta^{2}\left(\frac{\hbar \omega_{B}}{2}\right)^{2} \operatorname{csch}^{2}\left(\frac{\beta \hbar \omega_{B}}{2}\right)
$$

where $\beta=\frac{1}{k_{B} T}$. First, we highlight that Equation (25) imply as natural consequence that the entropy contains the degeneracy terms, due to relation $S=\frac{1}{T}(E-F)$. It is in fact due to the structure of von Neumann entropy, because the probability coefficients contain the information of the degeneracy factor. For example, in thermal equilibrium, this coefficient takes the Boltzmann form, so

$$
P_{n}(\xi)=[Z(\xi, T)]^{-1} g(\xi) e^{-\frac{\mathcal{E}_{n}(\xi)}{k_{B} T}}
$$

An opposite case occurs for the expected value of energy and the specific heat at constant field because these two physical quantities are obtained as the derivative in the temperature of the partition function.

To clarify the importance of the degeneracy, we analyze the following case. Instead of the term $\frac{g(B)}{2}$ appearing in Equations (25) and (27), we put a factor one, corresponding to treat a single oscillator, and we call the entropy for that case just $S(T, B)$. It is easy to show that the dependence of the magnetic field on the temperature for the isoentropic trajectories in the non-degenerate scenario obeys the proportionality $B \propto T$. This trivial relation gives us the possibility to obtain the relations between the temperatures along the cycle given by $\frac{T_{1}}{T_{2}}=\frac{T_{3}}{T_{4}}$, and the efficiency is reduced to a very well-known expression

$$
\eta=1-\frac{\omega\left(B_{1}\right)}{\omega\left(B_{2}\right)}
$$

which can be rewritten as

$$
\eta=1-\frac{1}{\left(\frac{l_{B_{1}}}{l_{B_{2}}}\right)^{2}} \equiv 1-\frac{1}{r^{3-1}},
$$

and we get the result $\gamma=3$, as described in the work of Zheng and Poletti [36].

\section{Results and Discussion}

For the Landau case, it is useful to rewrite the term of the degeneracy factor in the entropy as $\frac{g}{2}=\frac{\Phi(B)}{2 \Phi_{0}}$, where $\Phi(B)$ is the total magnetic flux and $\Phi_{0}$ is the universal quantum of magnetic flux, given by $h / 2 e$. Moreover, we define this degeneracy term in the entropy as $\frac{g}{2}=\lambda B$, where $\lambda=\frac{A}{2 \Phi_{0}}$. Thus, the entropy for this case given by Equation (27) depends on three variables, $S_{L} \equiv S_{L}(T, B, \lambda)$.

If the dependence of magnetic field and temperature in the adiabatic process for the Landau case is analyzed, we clearly see that the condition for entropy $S_{L}\left(T_{0}, B_{0}, \lambda\right)=S_{L}(T, B, \lambda)$ yields a relation between the magnetic field and temperature which will not depend on $\lambda$. This is because the degeneracy term $g(B)$ is associated with a logarithmic term, so the degeneracy effect in the cycle is only reflected in the magnetic field dependence of $g(B)$. To reinforce this idea, we calculate the structure of 
first order differential equation for the adiabatic processes through Equation (21), which for this case has the form

$$
\frac{d B}{d T}=-\frac{C_{1}^{2} \frac{B^{2}}{T^{3}} \operatorname{csch}^{2}\left(C_{1} \frac{B}{T}\right)}{\frac{1}{B}-C_{1}^{2} \frac{B}{T^{2}} \operatorname{csch}^{2}\left(C_{1} \frac{B}{T}\right)},
$$

where $C_{1}$ is a constant given by $C_{1}=\frac{e \hbar}{2 k_{B} m}$. This previous equation has an analytical solution (see Appendix A for details) given by

$$
C_{1} \frac{B}{T} \operatorname{coth}\left(C_{1} \frac{B}{T}\right)+\ln \left(C_{1} B\right)-\ln \left[\sinh \left(C_{1} \frac{B}{T}\right)\right]=\mathcal{C}_{2}
$$

where $\mathcal{C}_{2}$ is an integration constant. Note that the additional term in the differential equation which provides $g(B)$, is the factor $(1 / B)$ in the dominator of Equation (32). If this term does not exist, the differential equation has a simple form $\frac{d B}{d T}=\frac{B}{T}$ and obtains the result previously discussed for the non-degenerate case.

In Figure 2, we see the behavior of the magnetic field versus the temperature along an isoentropic trajectory, showing the linear dependence between the magnetic field and the temperature in the case of $g=1$ (non-degenerate) and for the case of high degeneracy. In order to see the scale of entropy for $S_{L}(T, B, \lambda)$ for real values, we select $\lambda \propto 10^{8} \mathrm{~T}^{-1}$, which means an active area of $A \propto 10^{-7} \mathrm{~m}^{2}$, by using the fact that the universal flux quantum has an order of $\Phi_{0} \propto 10^{-15} \mathrm{~Wb}$. In the left panel of Figure 2, we plot the solution for the case $S(T, B)=S(10,1)$, and in the right panel we plot the solution for the case $S\left(T, B, 10^{8}\right)=S\left(10,1,10^{8}\right)$. The contrast is evident: in the simple scenario an increase in the magnetic field implies an increase in the temperature. However, for the case with degeneracy, the rise in the magnetic field leads to a decrease in the temperature. The explanation of this fact lies in the behavior of the entropy at low temperatures, because of $S(T, B, \lambda)_{T \rightarrow 0} \sim k_{B} \ln (g)$, where $g$ is directly proportional to $B$. This is discussed in Figure 3 where we show the entropy behavior in these two different scenarios. In the non-degenerate case, when we increase the magnetic field, the function $S(T, B)$ intersects the starting value of the entropy always in a higher value than the initial one, reflected in the left frame of Figure 3. This explains the linearity that we obtain in a plot $B$ vs. $T$ for the left panel of Figure 2. The opposite occurs for the degenerate case, the function $S_{L}\left(T, B, 10^{8}\right)$, which intersects the starting value of the entropy always in a lower value than the initial one, as we see in the right frame of Figure 3. From this same figure, we can conclude that the entropy function for the degenerate case collapses to approximately the same value for higher temperature for different values of the magnetic field. Therefore, since the magnetic field is the external parameter that makes the engine work, we have a region of temperature and magnetic field where it is valid to carry out this cycle.
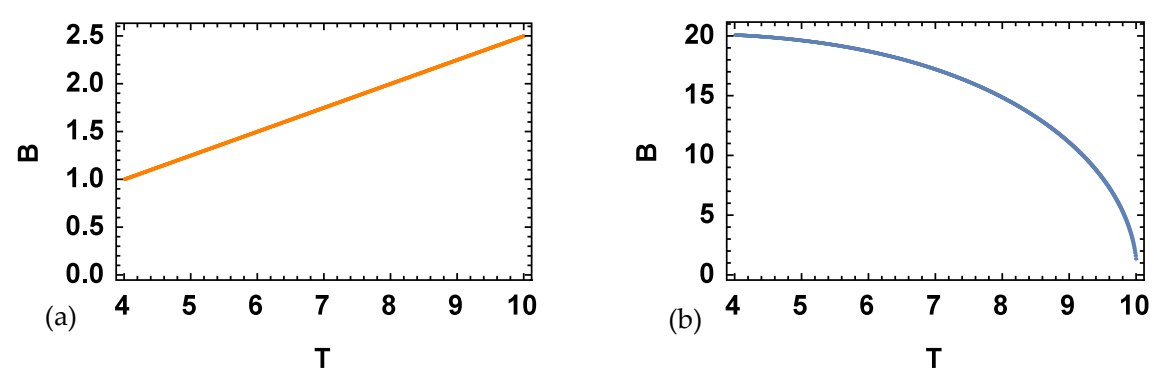

Figure 2. Behavior of the magnetic field versus the temperature for the case without the degeneracy factor (a) and the case with the degeneracy factor $\frac{\Phi(B)}{2 \Phi_{0}}(\mathbf{b})$. We select the factor $\frac{A}{2 \Phi_{0}} \propto 10^{8} \mathrm{~T}^{-1}$ for this example. 

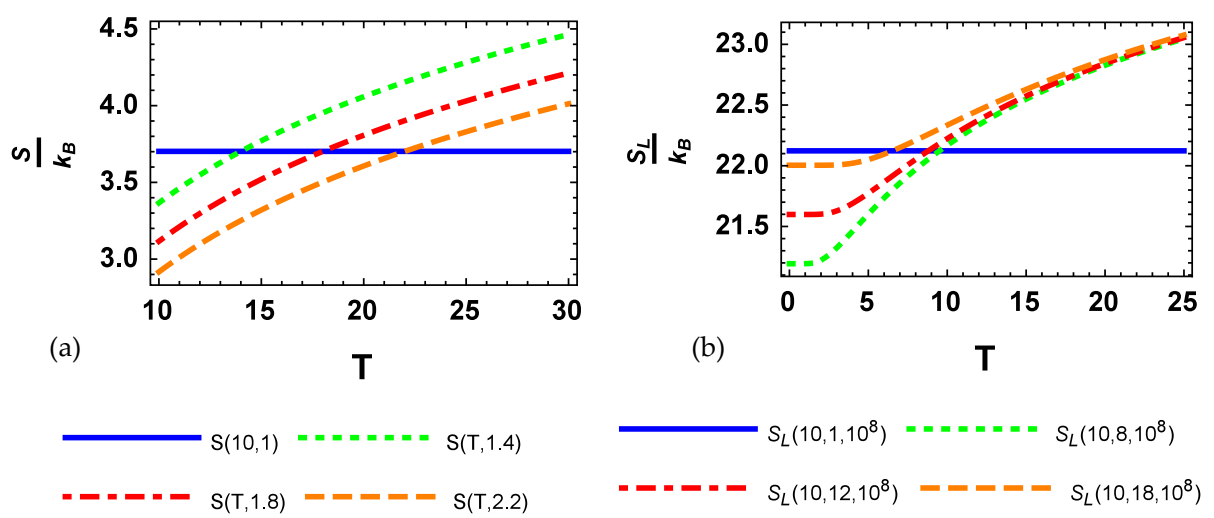

Figure 3. The isoentropic trajectories behavior for the two cases under discussion. In (a) we plot the non-degenerate case $S(T, B)=S(10,1)$ and in $(\mathbf{b})$ the degenerate case $S_{L}\left(T, B, 10^{8}\right)=S_{L}\left(10,1,10^{8}\right)$.

As discussed in Appendix A, with an adequate analysis of the asymptotic behaviours of Equation (32), we found a critical temperature, given by

$$
T_{\mathcal{C}}=e^{\left(\mathcal{C}_{2}-1\right)},
$$

which corresponds to the value of the temperature when the magnetic field goes to zero and a critical value for the magnetic field when it starts to become constant, given by the expression

$$
B_{c}=\frac{e^{\mathcal{C}_{2}}}{2 C_{1}}
$$

Therefore, we have the two points for an initial value constant $\mathcal{C}_{2}$ where it makes sense to carry out the cycle. For the exponential form of Equation (35), the critical value of the constant magnetic field is always a large quantity. For a real example of a starting field and temperature, we can consider the example of Figure 2, where the initial values of the intensity field and temperature are $1 \mathrm{~T}$ and a $10 \mathrm{~K}$, respectively. The approximate value for the critical values are $T_{c} \approx 10.1 \mathrm{~K}$ and $B_{c} \approx 20.1 \mathrm{~T}$.

For the starting point previously indicated, we can consider a cycle for the degenerate case like that in Figure 1 operating between the temperatures $4 \mathrm{~K}$ and $10 \mathrm{~K}$. However, due to the behavior of temperature along the adiabatic trajectory described in Figure 2, initially we brought the system into thermal equilibrium at $T_{1}>T_{3}$. Thus, for that case, the heat defined by $Q_{4 \rightarrow 1}$ corresponds to the heat absorbed, and for the heat released the correct definition is given by $Q_{2 \rightarrow 3}$, contrary to the non-degenerate case. To reinforce this idea, we display in the right frame of Figure 4 the behavior of heat along the cycle for the degenerate case and non-degenerate case. The convention of the sign (positive for heat absorbed) is satisfied along the entire operation of the engine. The positive work condition, which plays an essential role for a well defined thermal engine, is shown in the left frame of Figure 4 for both cases. From the same figure, we can extract relevant information about the $r$ parameter. For a machine operating between two reservoirs of $4 \mathrm{~K}$ and $10 \mathrm{~K}$, we obtain

$$
r_{\text {non-deg }}^{\max }=1.58 \text { and } \quad r_{\text {deg }}^{\max }=4.47,
$$

which represents the maximum value that can be taken for the compression ratio along the cycle and corresponds to the point where the Carnot efficiency is obtained. These results are natural only to see Figure 3 for this example. For the non-degenerate case, it is only necessary to increase the field by a factor of 2.5, but for the degenerate case it is necessary to increase the field by a factor of 20 to reach the Carnot efficiency of the problem. However, the two points previously discussed have the average work and power output equal to zero, as we see in the left panel of Figure 4 and the purpose 
of showing them is only conceptual. From Figure 4 we observe that the two points for the maximum power output in the two different scenarios under discussion are given by

$$
r_{\text {non-deg }}^{W} \approx 1.25 \text { and } r_{\text {deg }}^{W} \approx 3.66,
$$

with efficiency values $\eta\left(r_{\text {non-deg }}^{W}\right) \approx 0.34$ and $\eta\left(r_{\text {deg }}^{W}\right) \approx 0.26$, as we see in Figure 5. This combination of values is the really important in our problem because it corresponds to the optimal operating region of the proposed thermal machine.
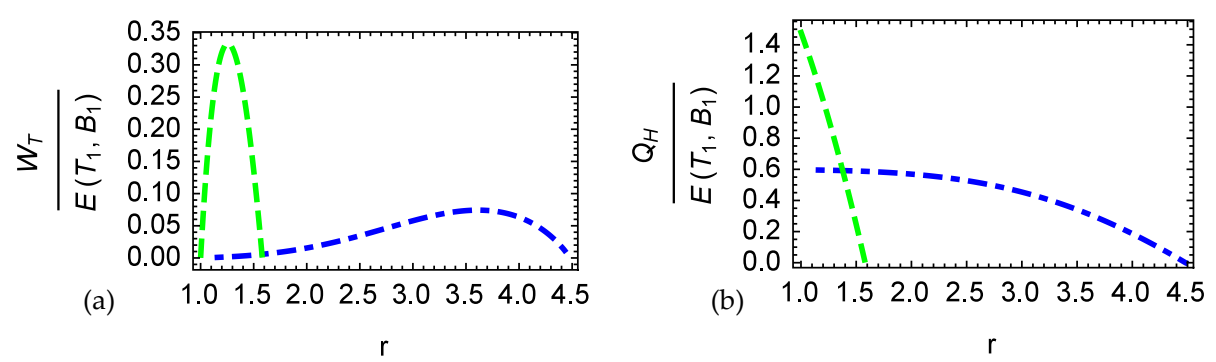

Figure 4. Total work (a) and input heat (b) versus the $r$ parameter along the cycle for the case with degeneracy (dot dashed line) and without degeneracy (dashed line).

In Figure 5 we compare the three efficiencies, where we see the effect of the degeneracy. One form to understand this behavior corresponds to the approximation that we show in Appendix A for the parametric solution, with the finality to "uncouple" the solution to magnetic field and the temperature for the adiabatic trajectory getting a function in the form

$$
B(T)=\frac{k}{2 C_{1}}\left(1-\mathrm{e}^{-k \sqrt{\frac{0.64}{T^{2}}\left(1-\mathrm{e}^{\frac{T}{k}}\right)}}\right),
$$

where we define $k=\mathrm{e}^{\mathcal{C}_{2}}$. Therefore, for this exponential form for the field as a function of temperature, when we parametrize the efficiency vs. a function of the typical compression ratio $(r)$, we obtain the behavior presented in Figure 5.

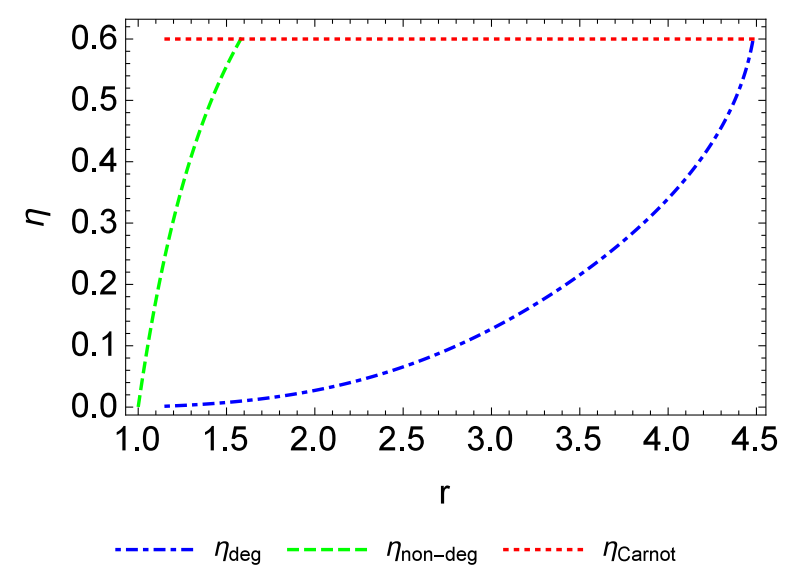

Figure 5. Efficiency for different cases of interest. For this case, the dotted red line corresponds to the value of Carnot cycle for a machine operating between the two temperatures $T_{1}=4 \mathrm{~K}$ and $T_{3}=10 \mathrm{~K}$.

For the definition of work and its interpretation as $W=-\int M d B$, we study the magnetization along the cycle defined as

$$
M=\frac{e \hbar}{2 m}\left(\frac{2}{\beta \hbar \omega_{B}}-\operatorname{coth}\left(\frac{\beta \hbar \omega_{B}}{2}\right)\right)
$$


For the adiabatic trajectory, the temperature and the magnetic field change along the entire process, so we can use a contour plot to see the value of magnetization displayed in Figure 6. Here, we clearly see that the values of magnetization are always negative and the same occurs for the different curves in the "isomagnetic" process, shown in Figure 7, indicating that the response of the system is diamagnetic.

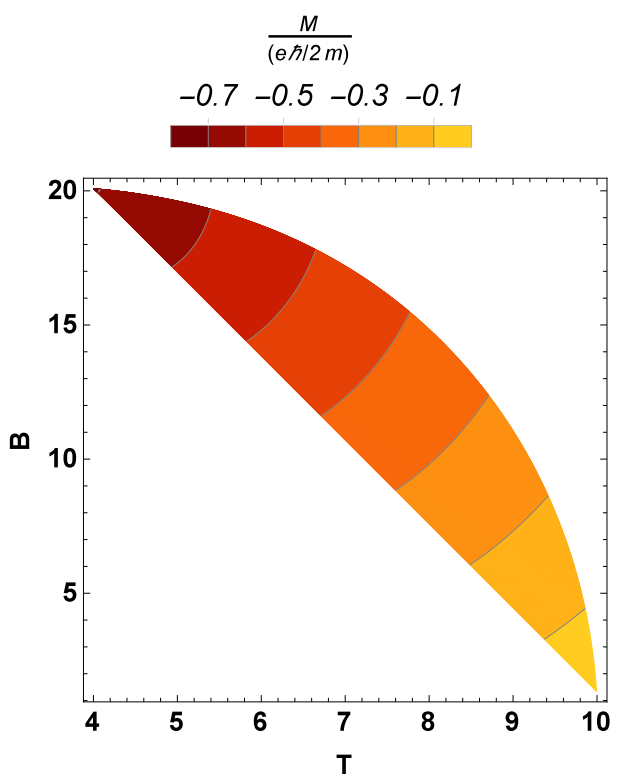

Figure 6. Magnetization as a function of $B$ and $T$ along the adiabatic trajectories.

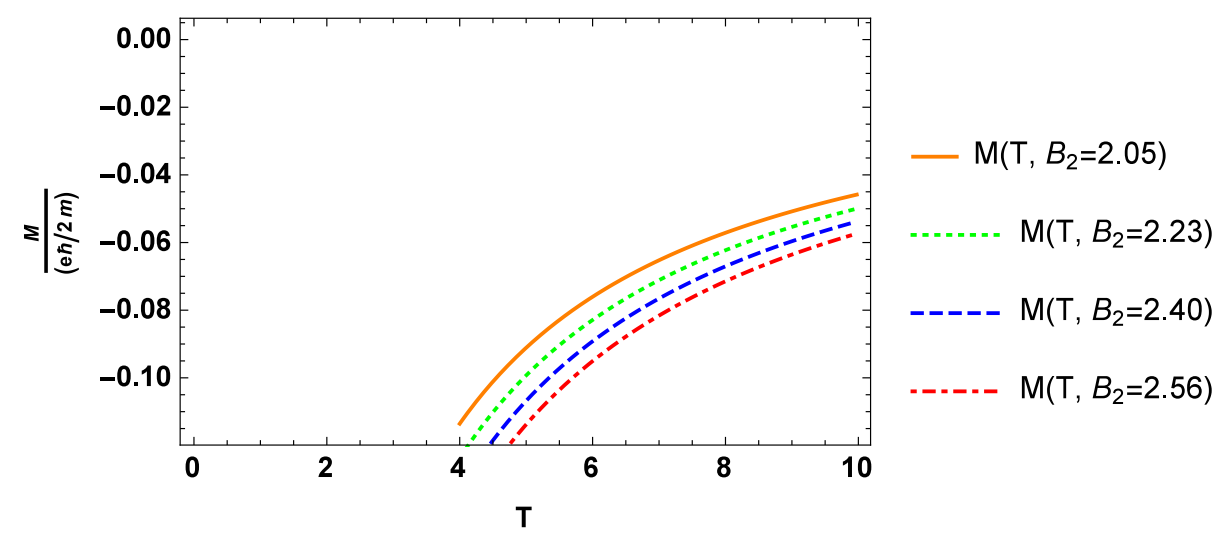

Figure 7. Magnetization along the first iso-magnetic trajectory as a function of $T$ in the range of $4 \mathrm{~K}$ to $10 \mathrm{~K}$. We selected the different values for $B_{2}$ that we found from numerical calculations.

Our system was studied to prove a concept rather than a practical implementation protocol. However, we believe the readers will find attractive the study of the "full" quantum version of this cycle following the treatment of the works [28-30], where treated the magnetic substance under degenerate conditions using non-equilibrium techniques. Besides, it is promising to treat the quantum version of our formulation optimized following the work of Kosloff and Rezek [25], for the case of frictionless adiabats using the methods of shortcuts to adiabaticity [37-39]. Moreover, this problem can be extended taking in account the edge states of the systems for a more realistic approach. Additionally, it is important to note that the regime of validity of this semi-classical approach deserves further investigation. 


\section{Conclusions}

In this work, we explored the possibility of constructing a single-particle magnetic engine of the Landau problem. In particular, we found an analytical solution for the dependence of the magnetic field and temperature in the adiabatic trajectories. We used this relation to obtain the form of the efficiency showing a radically different behavior of the typical harmonic case and found that a major increase in the external magnetic field to reach the Carnot efficiency is necessary. We remark that the useful work of this engine, related to change in the magnetization along the process, can be used for example in the generation of induction current in other physical systems.

It is important to note that our one-particle approach must be refined to take into account a many electrons scenario, which yields more precise calculations. However, the one electron case is important due to simplicity and the arising of richer physics for comparatives cases. For example, we can work with a one-particle system combining the effects of a cylindrical potential well, which physically represents an accurate model for a semiconductor quantum dot, and an externally imposed magnetic field, where the number of electrons can be controlled without problems; thus, the same analysis presented in this work can be replicated.

Acknowledgments: Francisco J. Peña acknowledges the financial support of FONDECYT-postdoctoral 3170010, as well as G. Alvarado Barrios and F. Albarrán-Arriagada for the important comments to improve the manuscript. Alvaro S. Nunez and Patricio Vargasv acknowledge support from Financiamiento Basal para Centros Científicos y Tecnológicos de Excelencia, under Project No. FB 0807 (Chile), Patricio Vargas acknowledges USM-DGIIP grant number PI-M-17-3 (Chile) and Alvaro S. Nunez acknowledges funding from FONDECYT program under grant 1150072. The authors acknowledge the referees for their constructive comments on our work.

Author Contributions: Francisco J. Peña and Patricio Vargas conceived the idea and formulated the theory. Alejandro González built the computer program and edited figures. René G. Rojas solved the differential equation and their respective asymptotic analysis. Alvaro S. Nunez and Pedro A. Orellana contributed to discussions during the entire work and the corresponding editing of the same. Francisco J. Peña wrote the paper. All authors have read and approved the final manuscript.

Conflicts of Interest: The authors declare no conflict of interest.

\section{Appendix A}

We need to solve the differential equation in the form

$$
\frac{d B}{d T}=-\frac{C_{1}^{2} \frac{B^{2}}{T^{3}} \operatorname{csch}^{2}\left(C_{1} \frac{B}{T}\right)}{\frac{1}{B}-C_{1}^{2} \frac{B}{T^{2}} \operatorname{csch}^{2}\left(C_{1} \frac{B}{T}\right)} .
$$

We define the parameter $u=C_{1}\left(\frac{B}{T}\right)$. So, differentiating respect to $T$, we obtain

$$
\frac{d u}{d T}=-C_{1} \frac{B}{T^{2}}+\frac{C_{1}}{T} \frac{d B}{d T} .
$$

Collecting these two last equations, we obtain the first order differential equation in the $u$ parameter in the form

$$
\frac{d u}{d T}=\frac{u}{T\left(u^{2} \operatorname{csch}^{2}(u)-1\right)},
$$

which corresponds to a differential equation of separable variables that has a solution given by

$$
u \operatorname{coth}(u)+\ln (u)-\ln [\sinh (u)]+\ln (T)=\mathcal{C}_{2},
$$

where $\mathcal{C}_{2}$ is a constant of integration. We can compact this solution if we define the two variables 


$$
y=\frac{C_{1} B}{k} \quad x=\frac{T}{k}
$$

where $k$ is given by

$$
k=\mathrm{e}^{\mathcal{C}_{2}},
$$

with e as the Euler number. So, the solution takes the parametric form

$$
y=\mathrm{e}^{-u \operatorname{coth} u} \sinh u \quad x=\frac{1}{u} \mathrm{e}^{-u \operatorname{coth} u} \sinh u .
$$

The asymptotic behaviors of these solutions is very interesting. The expression in the case of $u<<1$, which corresponds to high-temperature or small magnetic field limit, takes the form

$$
y=\frac{1}{\mathrm{e}} \sqrt{6(1-\mathrm{e} x)},
$$

so, we have a critical value, $x_{c}$, when $y \rightarrow 0$ given by

$$
x_{c}=\frac{1}{\mathrm{e}} .
$$

It gives us a critical temperature $T_{c}$ when the magnetic field goes to zero, and is strongly dependent on initial values of the problem under study, given by

$$
T_{c}=\frac{k}{\mathrm{e}} \equiv \mathrm{e}^{\left(\mathcal{C}_{2}-1\right)} .
$$

In the other case, for $u>>1$, which corresponds to low temperature or high magnetic field limit, we obtain

$$
y_{c}=\frac{1}{2}
$$

and, this represents a critical constant value for the magnetic field, given by

$$
B_{c}=\frac{k}{2 C_{1}} \equiv \frac{\mathrm{e}^{\mathcal{C}_{2}}}{2 C_{1}},
$$

Thus, it is important to keep in mind that, when we consider a variation of the magnetic field as the cause for effective work in the system, the limits discussed before impose physical variable restrictions to operate the quantum machine proposed in the text.

To understand the magnetic field behavior in an explicit form along the adiabatic process, we propose an approximated curve in the form

$$
y=\frac{1}{2}\left(1-\mathrm{e}^{\frac{-\sqrt{0.64(1-\mathrm{e} x)}}{x}}\right) .
$$

The exact parametric solution, the asymptotic behavior for the limiting cases $(u>>1$ and $u<<1)$ and our proposal function are displayed in Figure A1. 


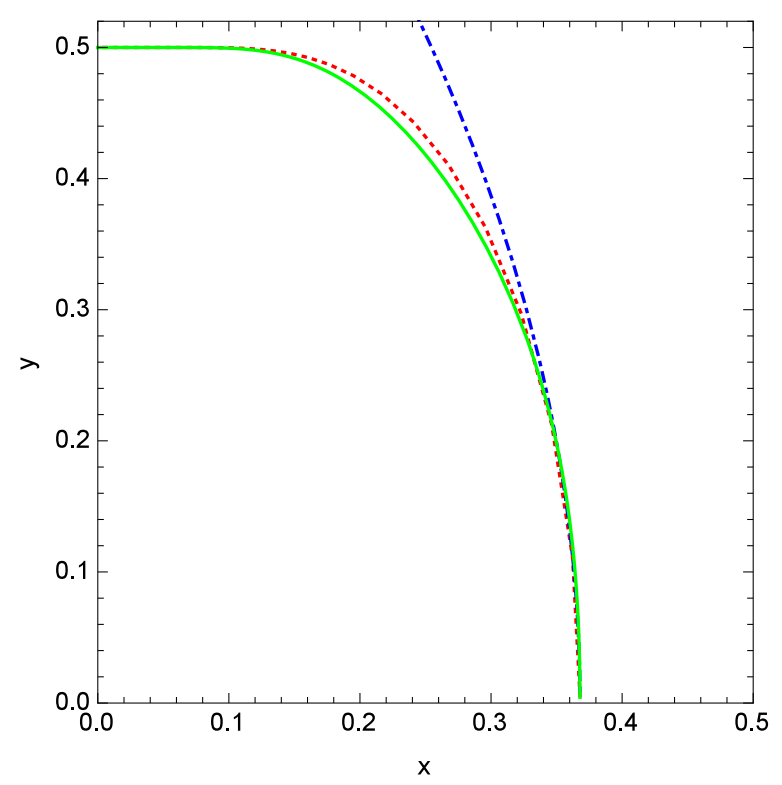

Figure A1. A parametric solution of the differential equation along the adiabatic trajectories for the Landau case. The dotted line represents the exact solution and the dot-dashed line the asymptotic case for $u<<1$. We can clearly see the constant value 0.5 for the solution in the case of $u>>1$ from the dotted line in the figure. The solid line represents the proposal curve given by Equation (A13) showing a good fit for the problem under study.

\section{References}

1. Scovil, H.E.D.; Schulz-DuBois, D.O. Three-Level masers as a heat engines. Phys. Rev. Lett. 1959, 2, $262-263$.

2. Huang, X.L.; Niu, X.Y.; Xiu, X.M.; Yi, X.X. Quantum Stirling heat engine and refrigerator with single and coupled spin systems. Eur. Phys. J. D 2014, 68, doi:10.1140/epjd/e2013-40536-0.

3. Su, S.H.; Luo, X.Q.; Chen, J.C.; Sun, C.P. Angle-dependent quantum Otto heat engine based on coherent dipole-dipole coupling. EPL 2016, 115, 30002.

4. Liu, S.; Ou, C. Maximum Power Output of Quantum Heat Engine with Energy Bath. Entropy 2016, $18,205$.

5. Scully, M.O.; Zubairy, M.S.; Agarwal, G.S.; Walther, H. Extracting work from a single heath bath via vanishing quantum coherence. Science 2003, 299, 862-864.

6. Scully, M.O.; Zubairy, M.S.; Dorfmann, K.E.; Kim, M.B.; Svidzinsky, A. Quantum heat engine power can be increased by noise-induced coherence. Proc. Natl. Acad. Sci. USA 2011, 108, 15097-15100.

7. Bender, C.M.; Brody, D.C.; Meister, B.K. Quantum mechanical Carnot engine. J. Phys. A Math. Gen. 2000, 33, 4427-4436.

8. Bender, C.M.; Brody, D.C.; Meister, B.K. Entropy and temperature of quantum Carnot engine. Proc. R. Soc. Lond. A 2002, 458, 1519-1526.

9. Wang, J.H.; Wu, Z.Q.; He, J. Quantum Otto engine of a two-level atom with single-mode fields. Phys. Rev. E 2012, 85, 041148.

10. Huang, X.L.; Xu, H.; Niu, X.Y.; Fu, Y.D. A special entangled quantum heat engine based on the two-qubit Heisenberg XX model. Phys. Scr. 2013, 88, 065008.

11. Muñoz, E.; Peña, F.J. Quantum heat engine in the relativistic limit: The case of Dirac particle. Phys. Rev. E 2012, 86, 061108.

12. Muñoz, E.; Peña, F.J. Magnetically driven quantum heat engine. Phys. Rev. E 2014, 89, 052107.

13. Peña, F.J.; Muñoz, E. Magnetostrain-driven quantum heat engine on a graphene flake. Phys. Rev. E 2015, 91, 052152.

14. Peña, F.J.; Ferré, M.; Orellana, P.A.; Rojas, R.G.; Vargas, P. Optimization of a relativistic quantum mechanical engine. Phys. Rev. E 2016, 94, 022109.

15. Wang, J.; He, J.; He, X. Performance analysis of a two-state quantum heat engine working with a single-mode radiation field in a cavity. Phys. Rev. E 2011, 84, 041127. 
16. Abe, S. Maximum-power quantum-mechanical Carnot engine. Phys. Rev. E 2011, 83, 041117.

17. Wang, J.H.; He, J.Z. Optimization on a three-level heat engine working with two noninteracting fermions in a one-dimensional box trap. J. App. Phys. 2012, 111, 043505.

18. Wang, R.; Wang, J.; He, J.; Ma, Y. Performance of a multilevel quantum heat engine of an ideal N-particle Fermi system. Phys. Rev. E 2012, 86, 021133.

19. Callen, H.B. Thermodynamics and an Introduction to Thermostatistic; Jhon Wiley \& Sons: New York, NY, USA, 1985.

20. Tolman, R.C. The Principles of Statistical Mechanics; Oxford University Press: Oxford, UK, 1938.

21. Chotorlishvili, L.; Azimi, M.; Stagraczyński, S.; Toklikishvili, Z.; Schüler, M.; Berakdar, J. Superadiabatic quantum heat engine with a multiferroic working medium. Phys. Rev. E 2016, 94, 032116.

22. Azimi, M.; Chorotorlisvili, L.; Mishra, S.K.; Vekua, T.; Hübner, W.; Berakdar, J. Quantum Otto heat engine based on a multiferroic chain working substance. New J. Phys. 2014, 16, 063018.

23. Jaramillo, J.; Beau, M.; del Campo, A. Quantum supremacy of many-particle thermal machines. New J. Phys. 2016, 18, 075019.

24. Del Campo, A.; Goold, J.; Paternostro, M. More bang for your buck: Super-adiabatic quantum engines. Sci. Rep. 2017, 4, doi:10.1038/srep06208.

25. Kosloff, R.; Rezek, Y. The Quantum Harmonic Otto Cycle. Entropy 2017, 19, 136.

26. Roßnagel, J.; Dawkins, T.K.; Tolazzi, N.K.; Abah, O.; Lutz, E.; Kaler-Schmidt, F.; Singer, K. A single-atom heat engine. Science 2016, 352, 325-329.

27. Kumar, J.; Sreeram, P.A.; Dattagupta, S. Low-temperature thermodynamics in the context of dissipative diamagnetism. Phys. Rev. E 2009, 79, 021130.

28. Dong, C.D.; Lefkidis, G.; Hübner, W. Quantum Isobaric Process in Ni2. J. Supercond. Nov. Magn. 2013, 26, 1589-1594.

29. Dong, C.D.; Lefkidis, G.; Hübner, W. Quantum Magnetic quantum diesel in Ni $i_{2}$. Phys. Rev. B 2013, 88, 214421.

30. Hübner, W.; Lefkidis, G.; Dong, C.D.; Chaudhuri, D. Spin-dependent Otto quantum heat engine based on a molecular substance. Phys. Rev. B 2014, 90, 024401.

31. Mehta, V.; Johal, R.S. Quantum Otto engine with exchange coupling in the presence of level degeneracy. Phys. Rev. E 2017, 96, 032110.

32. Mani, R.G.; Smet, J.H.; von Klitzing, K.; Narayanamurti, V.; Johnson, W.B.; Umansky, V. Zero-resistance states induced by electromagnetic-wave excitation in GaAs/AlGaAs heterostructures. Nature 2002, 420, 646-650.

33. Quan, H.T.; Zhang, P.; Sun, C.P. Quantum heat engine with multilevel quantum systems. Phys. Rev. E 2005, 72, 056110.

34. Muñoz, E.; Peña, F.J.; González, A. Magnetically-Driven Quantum Heat Engines: The Quasi-Static Limit of Their Efficiency. Entropy 2016, 18, 173.

35. Quan, H.T. Quantum thermodynamic cycles and quantum heat engines (II). Phys. Rev. E 2009, 79, 041129.

36. Zheng, Y.; Polleti, D. Work and efficiency of quantum Otto cycles in power-law trapping potentials. Phys. Rev. E 2014, 90, 012145.

37. Cui, Y.Y.; Chem, X.; Muga, J.G. Transient Particle Energies in Shortcuts to Adiabatic Expansions of Harmonic Traps. J. Phys. Chem. A 2016, 120, 2962-2969.

38. Beau, M.; Jaramillo, J.; del Campo, A. Scaling-up Quantum Heat Engines Efficiently via Shortcuts to Adiabaticity. Entropy 2016, 18, 168.

39. Deng, J.; Wang, Q.; Liu, Z.; Hänggi, P.; Gong, J. Boosting work characteristics and overall heat-engine performance via shortcuts to adibaticity: Quantum and classical systems. Phys. Rev. E 2013, 88, 062122.

(c) 2017 by the authors. Licensee MDPI, Basel, Switzerland. This article is an open access article distributed under the terms and conditions of the Creative Commons Attribution (CC BY) license (http:/ / creativecommons.org/licenses/by/4.0/). 